\title{
Topical delivery of silymarin constituents via the skin route
}

\author{
Chi-feng HUNG ${ }^{1}$, Yin-ku LIN², Li-wen ZHANG ${ }^{3}$, Ching-hsien CHANG ${ }^{2}$, Jia-you FANG ${ }^{3, *}$ \\ ${ }^{1}$ School of Medicine, Fu Jen Catholic University, Taipei County, Taiwan, China; ${ }^{2}$ Department of Traditional Chinese Medicine, Center \\ for Traditional Chinese Medicine, Chang Gung Memorial Hospital, Keelung, Taiwan, China; ${ }^{3}$ Pharmaceutics Laboratory, Graduate \\ Institute of Natural Products, Chang Gung University, Kweishan, Taoyuan, Taiwan, China
}

\begin{abstract}
Aim: Silibinin (SB), silydianin (SD), and silychristin (SC) are components of silymarin. These compounds can be used to protect the skin from oxidative stress induced by ultraviolet (UV) irradiation and treat it. To this end, the absorption of silymarin constituents via the skin was examined in the present report.

Methods: Transport of SB, SD, and SC under the same thermodynamic activity through and into the skin and the effects of pH were studied in vitro using a Franz diffusion assembly.

Results: The lipophilicity increased in the order of $\mathrm{SC}<\mathrm{SD}<\mathrm{SB}$. Increased lipophilicity of a compound resulted in higher skin deposition but had a minor effect on permeation across the skin in the less-ionized form ( $\mathrm{pH} \mathrm{8).} \mathrm{It} \mathrm{is} \mathrm{apparent} \mathrm{that} \mathrm{compounds} \mathrm{in} \mathrm{the} \mathrm{less-}$ ionized form showed higher skin uptake compared to the more-ionized form. Hyperproliferative skin produced by UVB exposure showed increased permeation of silymarin constituents in the less-ionized form, but it did not affect deposition within the skin. With in vivo topical application for 4 and $8 \mathrm{~h}$, the skin deposition of SB was higher than those of SD and SC by 3.5 4.0- and 30 40-fold, respectively. The skin disruption and erythema test demonstrated that the topical application of these compounds for up to $24 \mathrm{~h}$ caused no apparent skin irritation.

Conclusion: The basic profiles of silymarin permeation via skin route were established.
\end{abstract}

Keywords: silymarin; silibinin; skin; topical delivery; ultraviolet B; permeation; absorption

Acta Pharmacologica Sinica (2010) 31: 118-126; doi: 10.1038/aps.2009.186; published online 21 December 2009

\section{Introduction}

Exposure of skin to ultraviolet (UV) radiation results in a variety of biological effects, including inflammation, induction of oxidative stress, formation of sunburned cells, and immunologic alterations ${ }^{[1]}$. All of these play important roles in the development of non-melanoma skin cancer (NMSC), which is the most frequently diagnosed malignancy in Caucasians around the world ${ }^{[2,3]}$. Surgical and medical treatments of NMSC are difficult because of a high recurrence rate, the occurrence of multiple lesions, and location of tumors on the head, neck, and outer arms ${ }^{[4]}$. These limitations suggest the need for additional approaches to protect skin against UVcaused cellular damage and NMSC ${ }^{[5]}$.

Silymarin, a polyphenolic flavonoid isolated from seeds of the milk thistle [Silybum marianum (L) Gaertn], has been used for more than 2000 years as a traditional medicine to treat liver disorders and to protect the liver against poisoning from

\footnotetext{
* To whom correspondence should be addressed.

E-mail fajy@mail.cgu.edu.tw

Received 2009-09-28 Accepted 2009-11-20
}

chemical and environmental toxins ${ }^{[6]}$. Silymarin is composed primarily of silibinin (SB) together with small amounts of other stereoisomers, such as silydianin (SD) and silychristin (SC) (Figure 1$)^{[7]}$. The UV light that reaches the earth's surface comprises primarily UVA wavelengths (315-400 nm) and the remainder (approximately 5\%) contains the (295-320 nm) UVB radiation. Both UVA and UVB cause wavelength-dependent damage to human skin including skin cancer, whose incidence is dramatically increasing ${ }^{[8]}$. Recent studies showed that silymarin constituents could strongly protect against photocarcinogenesis and inhibit UVB and chemical tumor promoterinduced skin inflammation and edema ${ }^{[1,9-11]}$. Silymarin also attenuates UVA-induced damage to human keratinocytes ${ }^{[12]}$.

A previous study ${ }^{[13]}$ reported that the skin tissue distribution of silibinin is low by oral administration in mice. Hence topical delivery via the skin may be capable of attaining sufficient pharmacological activity by silymarin constituents. Suitable absorption is known to be an essential requirement for the satisfactory application of topical agents. Although silymarin and its components were demonstrated to have significant activity on UV-irradiated skin, there is no information on the 
<smiles>COc1cc([C@H]2Oc3cc([C@@H]4Oc5cc(O)cc(O)c5C(=O)[C@H]4O)ccc3O[C@H]2CO)ccc1O</smiles><smiles>COc1cc(C2C(=O)C3C2C=C([C@@H]2Oc4cc(O)cc(O)c4C(=O)[C@H]2O)C2COC3(O)C2)ccc1O</smiles><smiles>COc1cc(C2Oc3c(O)cc([C@@H]4Oc5cc(O)cc(O)c5C(=O)[C@H]4O)cc3C2CO)ccc1O</smiles>

Figure 1. Chemical structures of silibinin (SB), silydianin (SD), and silychristin (SC).

absorption and permeability parameters of these flavonoids in skin.

The aim of this work was to establish basic profiles of SB, $\mathrm{SD}$, and SC permeation via the skin. Another purpose was to investigate the correlation between skin absorption and physicochemical characteristics of these compounds. Both in vitro and in vivo skin absorption experiments were performed in this study. Possible pathways of the compounds via the skin were elucidated using skin treated by various strategies as permeation barriers. Moreover, UVB-irradiated skin was used as a skin barrier for permeation in order to mimic the clinical situation.

\section{Materials and methods Materials}

SB (CAS: 22888-70-6) was purchased from ChromaDex (Irvine, CA, USA). SD (CAS: 29782-68-1) was supplied by USP reference standards (Rockville, MD, USA). SC (CAS: 33889-69-9), a-terpineol, and oleic acid were obtained from Sigma-Aldrich (St Louis, MO, USA). All other chemicals and solvents were analytical grade and were used as received.

\section{Preparation of saturated solutions}

Saturated solutions of SB, SD, and SC were prepared in
$\mathrm{Na}_{2} \mathrm{HPO}_{4}$-citric acid buffer (Mcllvain buffer) with respective $\mathrm{pH}$ values of $6,8,9.9$, and 10.8. An excess amount of each compound was added to $1 \mathrm{~mL}$ of the selected buffers, and shaken reciprocally in an incubator at $37^{\circ} \mathrm{C}$ for $24 \mathrm{~h}$. The suspension was centrifuged at $10000 \mathrm{r} / \mathrm{min}$ for $10 \mathrm{~min}$, and the concentration of the compound in the supernatant was determined by high-performance liquid chromatography (HPLC) after an appropriate dilution.

\section{HPLC analytical method}

The HPLC system for silymarin constituents included an L-2130 pump, an L-2200 sample processor, and an L-2400 UVvisible detector all from Hitachi (Tokyo, Japan). A 25-cm-long, 4-mm inner diameter stainless RP-18 column (Merck, Darmstadt, Germany) was used as the stationary phase. The mobile phase was an acetonitrile-water (35:65) mixture at a flow rate of $1 \mathrm{~mL} / \mathrm{min}$. The UV-visible detector was set at $288 \mathrm{~nm}$. The $\log K^{\prime}$ value (capacity factor) of the compounds was determined isocratically using HPLC. The retention time of each compound was measured, and the $K^{\prime}$ value was calculated from the following equation:

$$
\log K^{\prime}=\lg \left[\left(t_{\mathrm{r}}-t_{0}\right) / t_{0}\right]
$$

where $t_{\mathrm{r}}$ is the retention time of each compound, and $t_{0}$ is the retention time of the non-retained solvent peak (methanol).

At the range $0.1-100 \mu \mathrm{g} / \mathrm{mL}$, the concentration of all silymarin constituents was linearly proportional to their chromatographic peak area. The limit of detection (LOD) of SB, $\mathrm{SD}$, and $\mathrm{SC}$ was determined to be $10 \mathrm{ng} / \mathrm{mL}, 15 \mathrm{ng} / \mathrm{mL}$, and $15 \mathrm{ng} / \mathrm{mL}$, respectively. The intra- and inter-assay precision and accuracy values were evaluated at the concentration range $0.1-100 \mu \mathrm{g} / \mathrm{mL}$. The overall precision, defined by the relative standard deviation (RSD), ranged from $0.9 \%$ to $7.2 \%$ on average. Analytical accuracy, expressed as the percentage difference between the mean of measured value and the known concentration, varied from $-5.3 \%$ to $7.1 \%$.

\section{Preparation of skin membranes}

Female nude mice ( 8 weeks old) were sacrificed, and full-thickness skin was excised from the dorsal region. To obtain delipidized skin, the stratum corneum side was pretreated with chloroform-methanol (2:1) for $1 \mathrm{~h}$. Five percent a-terpineol or oleic acid in a $25 \%$ ethanol/water vehicle was used to pretreat skin mounted on a Franz cell for $2 \mathrm{~h}$, followed by the in vitro skin absorption experiment.

To obtain UVB-irradiated skin, a Bio-Spectra System Illuminator (Vilber Lourmat, France) was used to emit UVB at a wavelength of $312 \mathrm{~nm}$. This method was modified from Moore et $a l^{[14]}$. Briefly, mice were exposed to a single UVB dose of $150 \mathrm{~mJ} / \mathrm{cm}^{2}$ for $7 \mathrm{~d}$ and killed after the last exposure. The distance between the UVB lamps and the dorsal skin was about $40 \mathrm{~cm}$. The morphology of the skin was verified by cyclooxygenase (COX)-2 and proliferating cell nuclear antigen (PCNA) staining. Each specimen was dehydrated using ethanol, embedded in paraffin wax, and stained with COX-2 or PCNA. For each skin sample, three different sites were examined and evaluated under light microscopy (Olympus IX70, 
Tokyo, Japan).

\section{In vitro skin absorption}

Skin with or without the various treatments was mounted on the receptor compartment of a Franz cell with the stratum corneum side facing upwards into the donor compartment. Five and a half milliliters of a 3: $7(v / v)$ ethanol-pH 7.4 buffer was used as the receptor medium to maintain the sink condition of the three compounds. The donor compartment was occluded by parafilm and filled with $0.5 \mathrm{~mL}$ of the vehicle containing flavonoids at a dose which ensured saturated solubility. The available diffusion area between the compartments was $0.785 \mathrm{~cm}^{2}$. The stirring rate and temperature were kept at 600 $\mathrm{r} / \mathrm{min}$ and $37^{\circ} \mathrm{C}$, respectively. At appropriate intervals, $300-\mu \mathrm{L}$ aliquots of the receptor medium were withdrawn and immediately replaced with an equal volume of fresh medium.

At the end of the in vitro experiment $(24 \mathrm{~h})$, the skin was removed from the cell and the skin surface was cleaned with a cotton wool swab immersed in water and methanol three times each. The skin was then weighed, cut with scissors, positioned in a glass homogenizer containing $1 \mathrm{~mL}$ of methanol, and homogenized for $10 \mathrm{~min}$ at $300 \mathrm{r} / \mathrm{min}$. The resulting solution was centrifuged for $10 \mathrm{~min}$ at $10000 \mathrm{r} / \mathrm{min}$ and then filtered through a polyvinylidene difluoride (PVDF) membrane with a pore size of $0.45 \mu \mathrm{m}$. All samples were analyzed by HPLC.

\section{In vivo skin absorption}

For the in vivo experiment, an 8-week-old nude mouse was used. A glass cylinder with an available area of $0.785 \mathrm{~cm}^{2}$ was placed on the dorsal skin with glue (Instant Super Glue ${ }^{\circledR}$, Kokuyo, Japan). An aliquot of $0.2 \mathrm{~mL}$ of vehicle with silymarin constituents was added to the cylinder. The application times of the vehicle were 4 and $8 \mathrm{~h}$. The application region of the skin was excised at the end of the experiment. The procedures for washing and extraction of the compound from the skin were the same as for the in vitro experiment.

\section{In vivo skin irritation test}

A 0.6-mL aliquot of $\mathrm{pH} 8$ buffer with flavonoids was spread uniformly over a sheet of non-woven polyethylene cloth (1.5 $\mathrm{cm} \times 1.5 \mathrm{~cm}$ ), which was then applied to the back area of a nude mouse. The polyethylene cloth was fixed with Tegaderm ${ }^{\circledR}$ adhesive dressing (3M, USA) and Fixomull ${ }^{\circledR}$ stretch adhesive tape (Beiersdorf AG, Germany). After $24 \mathrm{~h}$, the cloth was removed, and the treated skin area was swabbed clean with a cotton wool swab. After withdrawal of the vehicle for $30 \mathrm{~min}$, transepidermal water loss (TEWL), colorimetric parameters, and the $\mathrm{pH}$ of the applied skin were measured. TEWL was recorded using a Tewameter ${ }^{\circledR}$ (TM300, Courage \& Khazaka, Köln, Germany). Measurements taken at a stable level were performed $30 \mathrm{~s}$ after application of the TEWL probe to the skin. The TEWL was automatically calculated and expressed in $\mathrm{g} \cdot \mathrm{m}^{-2} \cdot \mathrm{h}^{-1}$. A spectrocolorimeter (CD100, Yokogawa Electrical, Tokyo, Japan) was used to measure the skin erythema $\left(a^{*}\right)$. The instrument records color reflectance three-dimensionally
$\left(\mathrm{L}^{*}, \mathrm{a}^{*}, \mathrm{~b}^{*}\right)$ as recommended by the CIE (Commission Internationale de l'Eclairage). When recording the color values, the measuring head was held perpendicular to the dorsal skin of the mouse, and the aperture was fitted with an applicator, to avoid compression of the subcutaneous capillaries. The reading was obtained within a few seconds on the display. The skin surface $\mathrm{pH}$ was determined by a Skin-pH-Meter ${ }^{\circledR} \mathrm{PH} 905$ (Courage \& Khazaka, Germany). An adjacent untreated site was used as a baseline standard for each determination. The temperature and relative humidity in the laboratory were kept at $26{ }^{\circ} \mathrm{C}$ and $55 \%$, respectively. The sample number for each experiment was six $(n=6)$.

\section{Data analysis}

To calculate the permeation parameters of Fick's law from the plot of the cumulative amount versus time, a graph was plotted as shown in Figure 2. The flux value at the steadystate was determined and expressed per unit of diffusion area in $\mu \mathrm{g} \cdot \mathrm{cm}^{-2} \cdot \mathrm{h}^{-1}$ by a linear regression calculation from the slope of the linear portion of the cumulative amount-time profiles. The permeability coefficient $\left(K_{\mathrm{p}}, \mathrm{cm} / \mathrm{h}\right)$ was calculated from the flux divided by the saturated compound concentration in the donor compartment. The compound amount in the skin $(\mu \mathrm{g} / \mathrm{g})$ was also calibrated by the saturated solubility $(\mu \mathrm{g} / \mathrm{mL})$ in the donor compartment (calibrated skin deposition) to compare the skin absorption among different silymarin constituents.

A statistical analysis of differences between different treatments was performed using unpaired Student's $t$-test. A 0.05 level of probability was taken as the level of significance. An analysis of variance (ANOVA) test was also used if necessary.

\section{Results}

\section{In vitro skin absorption of SB}

The effect of the $\mathrm{pH}$ of buffers on skin permeation of SB was examined from $\mathrm{pH} 6$ to 10.8. The ionization of these compounds increased following an increase in the $\mathrm{pH}$. The saturated solubilities of $\mathrm{SB}$ in $\mathrm{pH} 6,8,9.9$, and 10.8 buffers were $4.20 \pm 0.41,31.41 \pm 1.86,190.53 \pm 6.63$, and $565.10 \pm 24.64 \mu \mathrm{g} / \mathrm{mL}$, respectively. This indicates that the ionic form of SB provided a more-hydrophilic condition of molecules.

In vitro delivery of SB into/across excised skin was investigated for all buffers from $\mathrm{pH} 6$ to 10.8. The permeation characteristics of SB are summarized in Table 1. Figure 2 shows an example of an SB permeation profile to illustrate the permeation kinetics during $48 \mathrm{~h}$. Cumulative amount-time profiles demonstrated the attainment of a steady-state flux of SB across the skin. SB flux was greatest from the saturated solution at $\mathrm{pH} 10.8$ and least from that at $\mathrm{pH}$ 6. This trend corresponded to the trend in the concentrations of SB in different saturated solutions. When calibrated by the saturated solubility $\left(K_{\mathrm{p}}\right)$, the SB permeability from $\mathrm{pH} 6$ buffer showed the greatest level, followed by $\mathrm{pH} 10.8,9.9$, and 8 . For topical formulations, the drug skin content is considered an important parameter. In the present work, the skin deposition was determined at the end of the in vitro experiments as depicted in Table 1 . The 
Table 1. Permeation data of $\mathrm{SB}$ from aqueous solutions with various $\mathrm{pH}$ values. $n=4$. Mean \pm SD.

\begin{tabular}{lccc}
\hline Vehicle & $\begin{array}{c}\text { Flux } \\
\left(\mu \mathrm{g} \cdot \mathrm{cm}^{-2} \cdot \mathrm{h}^{-1} \times 10^{-3}\right)\end{array}$ & $\begin{array}{c}\mathrm{K}_{\mathrm{p}} \\
\left(\mathrm{cm} / \mathrm{h} \times 10^{-3}\right)^{\mathrm{a}}\end{array}$ & $\begin{array}{c}\text { Calibrated } \\
\text { skin deposition } \\
\left(\times 10^{-1}\right)^{\mathrm{b}}\end{array}$ \\
\hline pH 6 buffer & $10.92 \pm 4.20$ & $2.60 \pm 1.00$ & $9.35 \pm 0.91$ \\
pH 8 buffer & $48.18 \pm 13.02$ & $0.74 \pm 0.20$ & $8.31 \pm 1.09$ \\
pH 9.9 buffer & $209.58 \pm 43.82$ & $1.10 \pm 0.23$ & $0.78 \pm 0.19$ \\
pH 10.8 buffer & $858.95 \pm 124.32$ & $1.52 \pm 0.22$ & $0.17 \pm 0.02$ \\
\hline
\end{tabular}

${ }^{a} K_{p}$, Permeability coefficient=Flux $\left(\mu g \cdot \mathrm{cm}^{-2} \cdot h^{-1}\right) /$ solubility $(\mu \mathrm{g} / \mathrm{mL})$.

${ }^{\mathrm{b}}$ Calibrated skin deposition=compound amount retained in skin $(\mu \mathrm{g} / \mathrm{g}) /$ solubility $(\mu \mathrm{g} / \mathrm{mL})$.

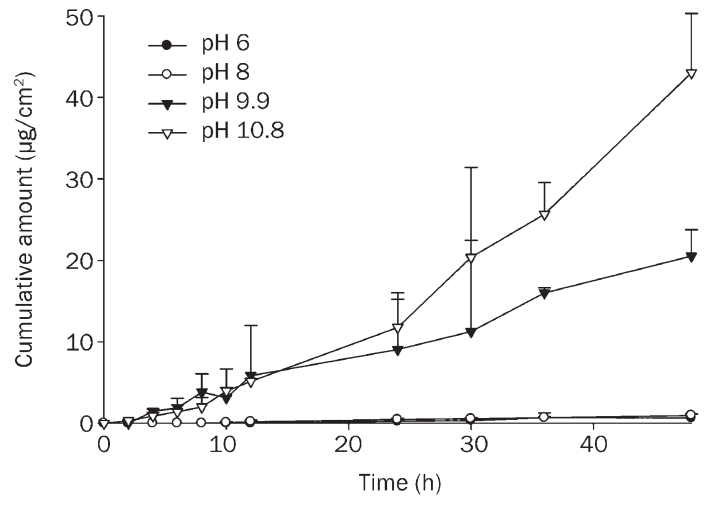

Figure 2. Cumulative amount versus time profiles of in vitro topical silibinin (SB) application permeating across nude mouse skin from aqueous buffers with different $p H$ values. $n=4$. Mean \pm SD.

deposition of SB was noted to have an increasing trend as the $\mathrm{pH}$ of the donor compartment decreased. The calibrated skin deposition from $\mathrm{pH} 6$ buffer exhibited a 55-fold increase compared to that from $\mathrm{pH} 10.8$ buffer.

\section{In vitro skin permeation of SB across various skin types}

Although the examination of drug permeability across the skin is less advantageous when targeting skin tissue, an understanding of the permeability may be helpful to elucidate the mechanisms involved in the skin absorption of the drugs. Table 2 summarizes the $K_{\mathrm{p}}$ of SB across various skin membranes from $\mathrm{pH} 8$ and 9.9 buffers. The $K_{\mathrm{p}}$ of SB with $\mathrm{pH} 8$ buffer across delipidized skin showed a 2.15-fold $(P<0.05)$ increase compared to that of intact skin. On the other hand, the data indicated that the $K_{\mathrm{p}}$ of $\mathrm{SB}$ with $\mathrm{pH} 9.9$ buffer across delipidized skin was 11.46 -fold higher $(P<0.05)$ than that across intact skin. To further explore the permeation mechanisms of $\mathrm{SB}$, a-terpineol and oleic acid were used to pretreat the skin. Ethanol at $25 \%$ was used as the pretreatment medium for solubility considerations. Ethanol had no statistically significant effect $(P>0.05)$ on SB permeability at $\mathrm{pH} 8$. Similar $K_{\mathrm{p}}$ values $(P>0.05)$ were observed for permeation across skin treated with a-terpineol or oleic acid and $25 \%$ ethanol. Ethanol at $25 \%$
Table 2. Permeation data of $\mathrm{SB}$ from $\mathrm{pH} 8$ and $\mathrm{pH} 9.9$ aqueous solutions across various skin types. $n=4$. Mean $\pm S D$.

\begin{tabular}{|c|c|c|c|}
\hline $\mathrm{pH}$ value & Skin type & $\begin{array}{c}K_{\mathrm{p}} \\
\left(\mathrm{cm} / \mathrm{h} \times 10^{-3}\right)^{\mathrm{a}}\end{array}$ & $\begin{array}{l}\text { Enhancement } \\
\text { ratio }(E R)^{b}\end{array}$ \\
\hline \multirow[t]{5}{*}{ pH 8} & Intact skin & $0.74 \pm 0.20$ & - \\
\hline & Delipid skin & $1.59 \pm 0.15$ & 2.15 \\
\hline & $25 \%$ ethanol treatment & $0.93 \pm 0.47$ & 1.26 \\
\hline & $\alpha$-Terpineol treatment ${ }^{\circ}$ & $0.85 \pm 0.11$ & 1.15 \\
\hline & Oleic acid treatment ${ }^{c}$ & $1.06 \pm 0.34$ & 1.43 \\
\hline \multirow[t]{5}{*}{ pH 9.9} & Intact skin & $1.10 \pm 0.23$ & - \\
\hline & Delipid skin & $12.61 \pm 0.45$ & 11.46 \\
\hline & $25 \%$ ethanol treatment & $4.78 \pm 2.41$ & 4.35 \\
\hline & $\alpha$-Terpineol treatment ${ }^{c}$ & $12.49 \pm 0.48$ & 11.35 \\
\hline & Oleic acid treatment ${ }^{c}$ & $5.39 \pm 0.91$ & 4.90 \\
\hline
\end{tabular}

${ }^{a} K_{p}$, Permeability coefficient=Flux $\left(\mu \mathrm{g} \cdot \mathrm{cm}^{-2} \cdot \mathrm{h}^{-1}\right) /$ solubility $(\mu \mathrm{g} / \mathrm{mL})$.

${ }^{\mathrm{b}}$ Enhancement ratio (ER), $K_{\mathrm{p}}$ across treated skin $/ K_{\mathrm{p}}$ across intact skin.

${ }^{\mathrm{C}}$ The medium is $25 \%$ ethanol.

significantly increased $(P<0.05)$ the $K_{\mathrm{p}}$ of SB compared to the control by 4.35 -fold. The enhancement ratios (ERs) of $K_{\mathrm{p}}$ for a-terpineol and oleic acid were 11.35 and 4.90, respectively. This indicated that pretreatment of the skin with a-terpineol further increased the permeation of SB in a more-ionized form.

\section{Comparison of the in vitro skin absorption levels of SB, SD, and SC}

The aqueous solubility and lipophilicity profiles of SD and SC were established for comparison with those of SB as shown in Table 3. The solubility increased in the order of $\mathrm{SB}<\mathrm{SD}<\mathrm{SC}$ in both $\mathrm{pH} 8$ and 9.9 buffers. The lipophilicity ranking was evaluated by measuring the capacity factor $\left(\log K^{\prime}\right)$, which indicates the relative retention of a compound in the HPLC system. The $\log K^{\prime}$ result confirmed the greater lipophilicity of SB compared to SD and SC. Although there are similarities in the structures of silymarin constituents, the $K_{\mathrm{p}}$ and skin deposition of these compounds showed discrepancies as demonstrated in Figure 3. In the less-ionized form ( $\mathrm{pH} 8), \mathrm{SC}$ showed the highest $K_{\mathrm{p}}$, followed by SB and SD $(P<0.05)$. A similar trend was observed in the more-ionized form ( $\mathrm{pH} 9.9)$ although higher values were detected for $\mathrm{pH} 9.9$ compared to $\mathrm{pH} 8$. The in vitro skin uptake of $\mathrm{SB}$ at $\mathrm{pH} 8$ was $\sim 4$-fold higher than that of SD and SC into intact skin. A correlation

Table 3. Solubility $(\mu \mathrm{g} / \mathrm{mL})$ and capacity factor ( $\left.\log K^{\prime}\right)$ of SB, SD, and SC in various vehicles. $n=6$. Mean $\pm S D$.

\begin{tabular}{lccc}
\hline Compound & $\begin{array}{c}\text { Solubility in } \\
\text { pH 8 buffer }\end{array}$ & $\begin{array}{l}\text { Solubility in } \\
\text { pH 9.9 buffer }\end{array}$ & $\log K^{\text {a }}$ \\
\hline Silibinin (SB) & $31.41 \pm 1.86$ & $190.53 \pm 6.63$ & 0.80 \\
Silydianin (SD) & $91.08 \pm 22.71$ & $440.83 \pm 3.13$ & 0.45 \\
Silychristin (SC) & $187.89 \pm 12.75$ & $821.68 \pm 48.80$ & 0.41 \\
\hline
\end{tabular}

${ }^{a} \log K^{\prime}, \operatorname{logarithm}$ of $\left(t_{r}-t_{0}\right) / t_{0}, t_{\mathrm{r}}$ is the retention time of product peak, $t_{0}$ is the retention time of solvent peak. 
A
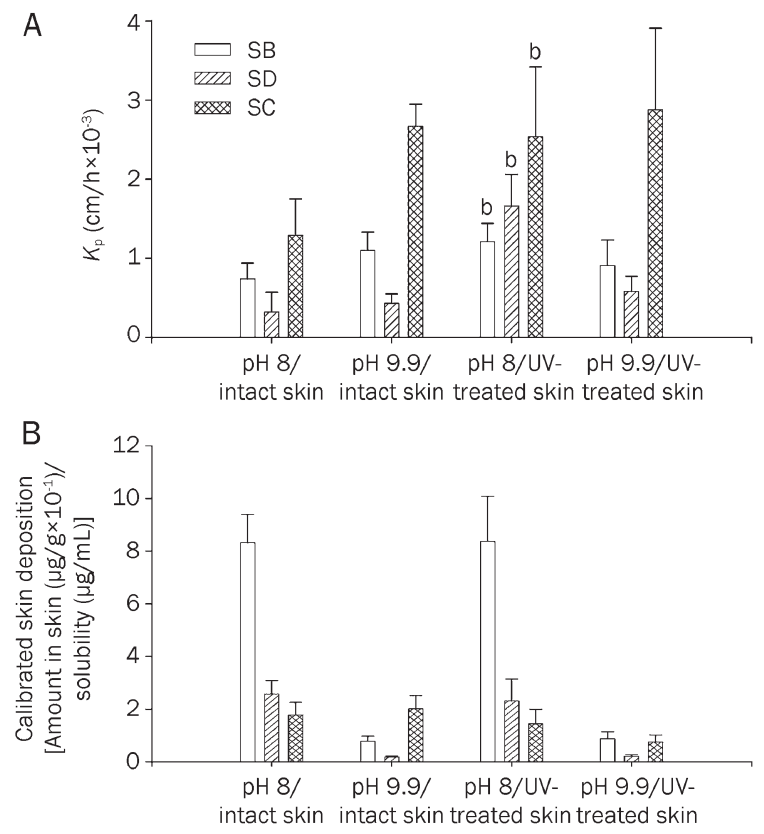

Figure 3 . The permeability coefficient $\left(K_{\mathrm{p}}\right)(\mathrm{A})$ and in vitro calibrated skin deposition (B) of silibinin (SB), silydianin (SD), and silychristin (SC) from $\mathrm{pH} 8$ and 9 buffers across intact or UVB-irradiated skin. $n=4$. Mean \pm SD. ${ }^{\mathrm{b}} \mathrm{P}<0.05$ vs $\mathrm{pH} 8 /$ intact skin.

was noted between $K_{\mathrm{p}}$ and skin retention of these compounds with pH 9.9 buffer.

\section{In vitro skin absorption across UVB-irradiated skin}

The appearance, histological morphology, and compound delivery of UVB-irradiated skin were examined. Figure 4A and $4 \mathrm{~B}$ depict representative examples of images of the skin surface without and with UVB exposure, respectively. UVB treatment was effective in inducing inflammatory responses including erythema and edema formation. The skin of mice was also desquamated after $7 \mathrm{~d}$ of UVB irradiation. As shown in Figure 4C and 4D, COX-2 expression was observed in epidermal cells of the dorsal skin of mice after UVB irradiation. Skin samples isolated from the treated area revealed an epidermal thickening in response to UVB-induced injury in these animals. The UVB irradiation increased the epidermal thickness by $\sim 5$-fold. Marked hyperplastic and hyperkeratotic changes with acanthosis were also detected. As shown in Figure $4 \mathrm{E}$ and $4 \mathrm{~F}$, the expression of PCNA was higher than without UVB irradiation. The PCNA results confirm cellular proliferation, reflecting the intense hyperproliferative process induced by $7 \mathrm{~d}$ of UVB exposure.

Figure 3 compares in vitro skin absorption of silymarin components via normal and hyperproliferative skin. UVBirradiated skin exhibited higher $K_{\mathrm{p}}$ values compared to normal skin $(P<0.05)$ in $\mathrm{pH} 8$ buffer. ER values with UVB treatment were $2.58,5.19$, and 1.97 for SB, SD, and SC, respectively. The more-ionized form ( $\mathrm{pH}$ 9.9) showed no significant differences $(P>0.05)$ between the two skin types. UVB irradiation did not influence the in vitro skin uptake of these compounds in either
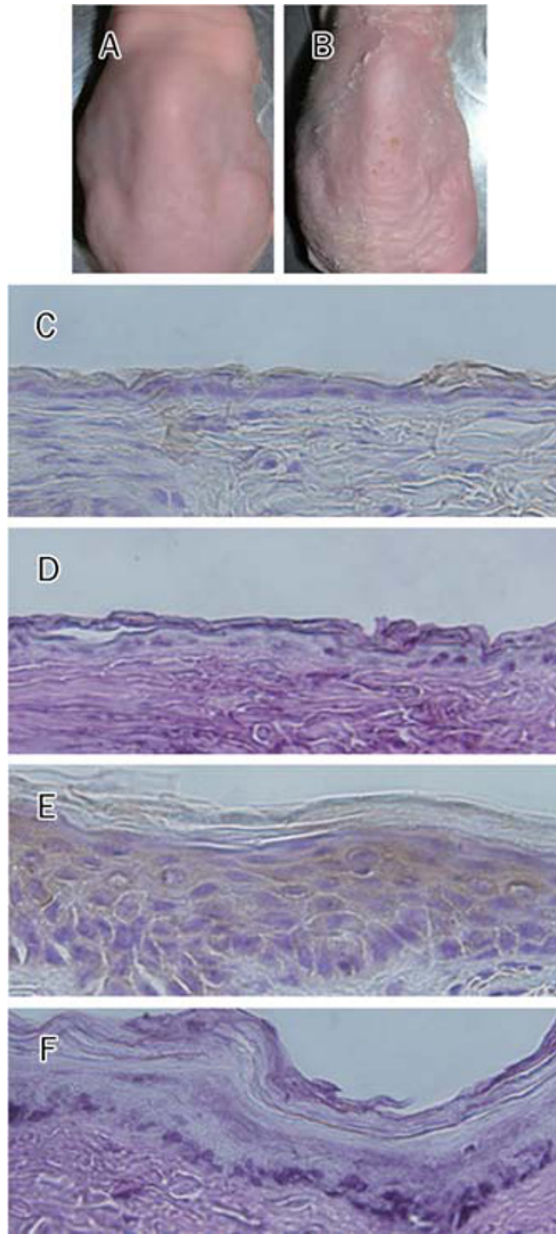

Figure 4. Images of nude mouse skin before and after UVB irradiation for $7 \mathrm{~d}$ : (A) clinical observation before UVB irradiation; (B) clinical observation after UVB irradiation; (C) COX-2 expression by immunochemical staining before UVB irradiation, $\times 400$; (D) COX-2 expression by immunochemical staining after UVB irradiation, $\times 400$; (E) PCNA expression by immunochemical staining before UVB irradiation, $\times 400$; (F) PCNA expression by immunochemical staining after UVB irradiation, $\times 400$.

pH 8 or 9.9 buffers $(P>0.05)$.

\section{In vivo skin absorption of SB, SD, and SC}

Levels of flavonoids in the skin were determined following a single application to the dorsal surface of nude mice. The $\mathrm{pH} 8$ buffer was selected as the vehicle because of the high compound accumulation within the skin in the in vitro status. Table 4 shows the in vivo skin deposition of SB, SD, and $\mathrm{SC}$ after topical delivery for 4 and $8 \mathrm{~h}$. Similar to the in vitro results, the in vivo uptake of SB was greater than those of SD and SC. There was no significant difference $(P>0.05)$ between the intradermal concentrations of any compound at 4 and $8 \mathrm{~h}$.

Bioengineering methods such as TEWL, colorimetry, and $\mathrm{pH}$ for evaluating the safety of silymarin constituents on skin were conducted in vivo. The $\Delta$ value (the value of the treated site minus the value of an adjacent untreated site) of TEWL, skin redness $\left(\mathrm{a}^{*}\right)$, and $\mathrm{pH}$ were determined after a 24-h admin- 
Table 4. In vivo skin deposition of SB, SD, and SC from $\mathrm{pH} 8$ aqueous solution. $n=6$. Mean \pm SD.

\begin{tabular}{lcc}
\hline Compound & $\begin{array}{c}\text { Calibrated skin deposition } \\
\text { at } 4 \mathrm{~h}\left(\times 10^{-1}\right)^{\mathrm{a}}\end{array}$ & $\begin{array}{c}\text { Calibrated skin deposition } \\
\text { at } 8 \mathrm{~h}\left(\times 10^{-1}\right)^{\mathrm{a}}\end{array}$ \\
\hline Silibinin (SB) & $8.07 \pm 1.93$ & $7.25 \pm 1.94$ \\
Silydianin (SD) & $2.11 \pm 0.25$ & $2.03 \pm 0.42$ \\
Silychristin (SC) & $0.20 \pm 0.04$ & $0.25 \pm 0.07$ \\
\hline
\end{tabular}

${ }^{a}$ Calibrated skin deposition=compound amount retained in skin $(\mu \mathrm{g} / \mathrm{g}) /$ solubility $(\mu \mathrm{g} / \mathrm{mL})$.

istration as shown in Figure 5. No significant skin irritation was determined when the bar of the standard deviation (SD) passes across the zero line in Figure 5. Silymarin constituents produced negligible changes in TEWL, suggesting a tolerance of the skin to topically applied formulations. Colorimetry measurements of $\mathrm{a}^{*}$ in nude mice did not change during the full treatment, indicating that erythema formation was not induced. Only a slight increment $(P<0.05)$ in the skin $\mathrm{pH}$ was observed in the case of SC.

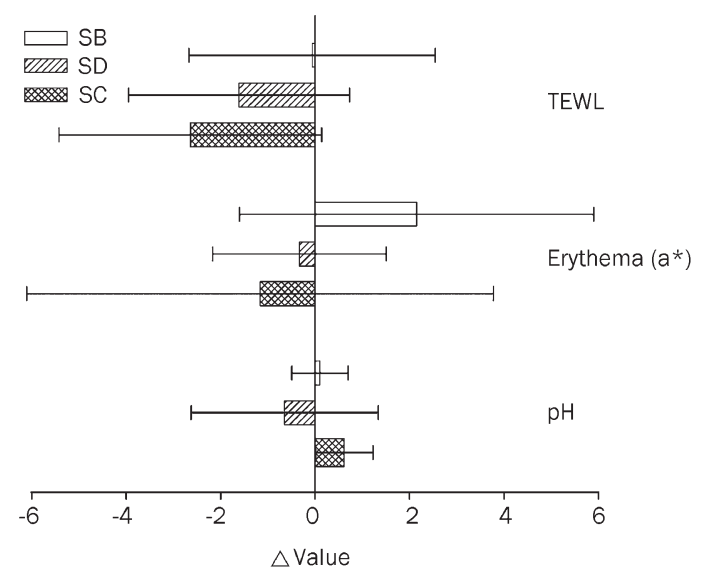

Figure 5. In vivo skin irritation examination determined by transepidermal water loss (TEWL), erythema $\left(a^{*}\right)$, and the $\mathrm{pH}$ value after a $24-\mathrm{h}$ application of topically applied silibinin (SB), silydianin (SD), and silychristin (SC) from $\mathrm{pH} 8$ buffer. The $\Delta$ value indicates the value of the treated site minus the value of an adjacent untreated site. $n=6$. Mean \pm SD.

\section{Discussion}

Studies focusing on the development of silymarin constituents with protective activity against UV-induced skin damage are intensively being carried out. Despite various reports linking the beneficial properties of silymarin to dermal use, no comprehensive study has been conducted investigating the skin absorption ability of these compounds. The purpose of this study was to identify formulation variables including $\mathrm{pH}$ and compounds that influence silymarin absorption via skin. We found that the structures of silymarin constituents largely affected their skin absorption. The results showed that
SB exhibited considerable absorption into the skin, especially in the non-ionized form. Negligible skin irritation was also detected.

SB was first selected to evaluate its skin absorption because SB is the most active and abundant constituent present in silymarin $^{[7]}$. In the skin absorption studies, the selection of a skin model is an important prerequisite. The most reliable skin absorption data are collected from human studies. However, such studies are generally not feasible during the initial development of a novel dosage form or system. The availability of such systems is also limited. The skin of rodents is most commonly used for in vitro and in vivo skin permeation studies. There are a number of hairless species (eg, nude mice and hairless rats) in which the absence of a hairy coat mimics the human skin better than hairy $\operatorname{skin}^{[15]}$. Hence nude mice were used as an animal model in this study. Although nude mouse skin is more permeable than human $\operatorname{skin}^{[16,17]}$, it is still a good model for examining the skin transport of permeants because of the limited variability among individuals and similar hair follicle density to human skin ${ }^{[18]}$.

Saturated solutions were used for the skin absorption experiment to ensure that there was uniform thermodynamic activity and thus common activity by the compounds in each formulation $^{[19,20]}$. The $\mathrm{pH}$ of the aqueous vehicle was shown to be one of the major variables that can influence the diffusivity of drugs ${ }^{[21,22]}$. The structures of silymarin constituents have multiple protonation sites. For example, the acidicdissociation constants of $\mathrm{SB}$ at $37{ }^{\circ} \mathrm{C}$ were determined to be $\mathrm{pK}_{\mathrm{a} 1}=6.86, \mathrm{pK}_{\mathrm{a} 2}=8.77, \mathrm{pK}_{\mathrm{a} 3}=9.62$, and $\mathrm{pK}_{\mathrm{a} 4}=11.38^{[23]}$. SB is predominantly in a non-ionic form in $\mathrm{pH} 6$ buffer, which is beneficial due to its lipophilicity. The neutral form of a compound always shows higher skin partitioning compared to the ionic form because of the lipophilic characteristics of the stratum corneum. The ionized degree of SB increased following an increase in the donor $\mathrm{pH}$ value. The results showed that the skin deposition of SB from an aqueous solution was directly related to its lipophilicity.

The higher skin reservoir of non-ionic SB may result in a high release into the receptor compartment because of the fast diffusion due to the concentration gradient. However, the trend of $K_{\mathrm{p}}$ values from $\mathrm{pH} 8$ to $\mathrm{pH} 10.8$ somewhat differed from that of calibrated skin deposition although there was no significant difference $(P>0.05)$ among $K_{\mathrm{p}}$ values from these three solutions. Increased $\mathrm{pH}$ can ionize a greater part of the intercellular fatty acids of the stratum corneum, changing the phase behavior and packing of the barrier lipid mixture ${ }^{[24]}$ A long-term application of an alkaline formulation on the skin surface might not be suitable for clinical use.

The predominant route for most permeant transport into or across the skin is the intercellular region of the stratum corneum. The delipidation process can remove intercellular lipids in the stratum corneum ${ }^{[20]}$. The stratum corneum is principally lipophilic in nature and far more resistant to polar than non-polar compounds. This speculation is consistent with the permeation profiles of SB with lipid removal which largely increased the permeation of the more-ionized form 
( $\mathrm{pH}$ 9.9) but not the less-ionized form ( $\mathrm{pH}$ 8). SB in $\mathrm{pH} 9.9$ buffer showed the highest enhancement of skin permeation across delipidized skin, suggesting that permeation through the stratum corneum layer was the rate-limiting process. The permeation of SB in $\mathrm{pH} 8$ buffer was not greatly enhanced by the extraction of lipid bilayers, or the main barrier to permeation could lie in the lower viable skin layer. Thus, it appears that the stratum corneum was not the sole significant contributor to resistance to the less-ionized form of SB. However, it cannot be neglected that this superficial layer still constitutes an important barrier for SB in $\mathrm{pH} 8$ buffer.

a-Terpineol and oleic acid are used as permeation enhancers for topical/transdermal drug delivery. Terpenes are known to act at the lipid polar heads of ceramides, while fatty acids act at the lipid tail portion of intercellular lipid bilayers ${ }^{[25,26]}$. Neither enhancer in $25 \%$ ethanol was able to express any enhancing activity on the permeation of SB in the less-ionized form after disruption of the lipid bilayers. This suggests that SB in $\mathrm{pH} 8$ buffer is easily partitioned into the bilayers. It also demonstrates that the intracellular route might not be important for lipophilic SB since oleic acid can affect corneocytes ${ }^{[27]}$.

Ethanol at 25\% increased SB permeation in $\mathrm{pH} 9.9$ buffer by 4.35 -fold. The ER by enhancers should be normalized to the value of $25 \%$ ethanol to neglect the vehicle influence. After calculation, it is found that a-terpineol but not oleic acid increased the permeation of the more-ionized form of SB. This result confirms that the lipid bilayers are the main barrier blocking the transit of ionic SB. Terpenes cause loosening of the lipid bilayers due to breaking of $\mathrm{H}$-bonds between ceramides as they have the potential to accept or donate $\mathrm{H}$-bonds ${ }^{[26]}$. The higher enhancement by a-terpineol than by oleic acid indicates that the diffusion of ionic SB along the lipid portion was more significant than that along the H-bonds between ceramides.

Skin absorption of a drug is determined by its physicochemical properties, in particular, the molecular weight and lipophilicity which play major roles in the process ${ }^{[20,28]}$. SB, $\mathrm{SD}$, and SC possess the same molecular weight of $482.4 \mathrm{Da}$. Therefore, any difference in skin absorption must have been attributable to another factor. There was a correlation between the skin deposition and lipophilicity of the three compounds in $\mathrm{pH} 8$ buffer. The highest lipophilicity of SB increased partitioning into the stratum corneum, forming a reservoir. On the other hand, SC has an additional hydroxyl group in its structure compared to SB and SD. The extra moiety contributes to the lower lipophilicity of SC. The amount of SC retained in the skin was also less because of its more-hydrophilic properties compared to the other compounds. However, lipophilicity is not a common rule for explaining the absorption trend of these flavonoids. SD generally showed the lowest values of $K_{\mathrm{p}}$ and deposition among the three compounds. A cyclic ketone moiety forming a steric bridge is seen in SD's structure (Figure 1). Both SB and SC show a planar structure. Although the molecular weights of these compounds are the same, SD may present a larger molecular volume compared to SB and $\mathrm{SC}$. This suggests evidence of the selective absorption of SD over SB and SC. In order to confirm this hypothesis, molecular modeling software (Discovery Studio ${ }^{\circledR}$ version 2.0, Accelrys Inc, San Diego, USA) was used to draw the stereo structure of the three flavonoids as shown in Figure 6. It is clearly seen that the three-dimensional structure of SD may hinder its penetration into skin. Further study is needed to explore the mechanisms involved in the relationship between the structure and skin absorption.

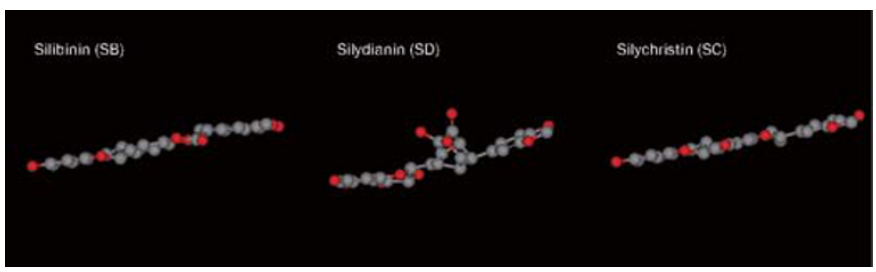

Figure 6. The stereo structure of silibinin (SB), silydianin (SD), and silychristin (SC) pictured by a molecular modeling software (Discovery Studio ${ }^{\circledR}$ version 2.0, Accelrys Inc, San Diego, USA).

It was expected that permeation through the skin would be reduced as the permeant is largely retained within the skin reservoir ${ }^{[29]}$. This theory can explain the contrary trend between $K_{\mathrm{p}}$ and skin deposition for the three flavonoids in the less-ionized form ( $\mathrm{pH} 8$ ). This phenomenon was not observed in $\mathrm{pH} 9.9$ buffer since a direct correlation between $K_{\mathrm{p}}$ and skin deposition was achieved for the more-ionized form. The concentration gradient between the skin and receptor may have contributed to this result. This suggests that the degree of ionization can influence the delivery behavior via the skin route.

UVB exposure can trigger oxidative damage and inflammation of the skin, subsequently inducing prominent epidermal thickening. Inflammation and DNA damage occur with UVB irradiation which lead to COX-2 and PCNA expressions ${ }^{[30,31]}$. PCNA expression is a marker of DNA repair and indirectly an indicator of UVB-induced damage ${ }^{[14]}$. These effects were confirmed by the histopathlogic profiles of skin tissue in the present study. When epidermal hyperplasia was well developed by UVB, $K_{\mathrm{p}}$ values of the flavonoids significantly increased in $\mathrm{pH} 8$ buffer. The increment in thickness of the epidermis may have created a longer pathway through which the permeant had to pass. This was not the case in the present study. The inflammation produced disruption of the epidermal structure. As indicated in the previous section, viable epidermis/dermis is an important barrier for neutral SB permeation. This disruption may attenuate the barrier function of viable skin, leading to increased $K_{\mathrm{p}}$ values. This result was not observed for ionized SB, indicating a limited alteration of lipid bilayers of the stratum corneum after UVB irradiation. Although permeability across the skin could be enhanced after exposure, retention within the skin reservoir remained unchanged. This result indicates that the dermal use of silymarin constituents for topical aims can minimize absorption differentiation between normal and disordered skin. Most research papers have utilized 
healthy skin to examine drug absorption, and results from such studies might not be appropriately applied to predict the skin targeting ability of a drug on disordered skin. The skin model used in this work may be useful for resolving this problem.

The in vivo topical application data show that silymarin constituents were efficiently absorbed into intact skin. A good in vitro-in vivo correlation was observed for the skin absorption trends from various formulations. This suggests that the amount of flavonoids retained in the skin in the in vitro status can predict the in vivo compound accumulation within the skin. According to a previous study by Lu et al ${ }^{[32]}$, the original contents of SB, SD, and SC in silymarin determined by HPLC were $33.40 \%, 3.51 \%$, and $12.91 \%$, respectively. It is beneficial for topical silymarin administration since SB, the compound with the highest percentage, showed greater skin absorption.

Besides the efficiency of diffusion into the skin, the skin tolerance is another concern for topical delivery systems. Many cutaneous reactions to herbal preparations and natural products have been reported ${ }^{[3,34]}$. The most common cutaneous adverse effect is allergic contact dermatitis. By evaluating established endpoints of skin irritation (TEWL, skin redness, and $\mathrm{pH}$ ), the present study demonstrates that the topical application of SB, SD, and SC for up to $24 \mathrm{~h}$ did not cause skin irritation. Oral administration and cell incubation of silymarin and the components have shown that they are well tolerated and do not cause any significant adverse health effects ${ }^{[6,12,35]}$. The same phenomenon was also observed in skin tissue.

\section{Conclusions}

The incidence of NMSC is directly associated with exposure to solar UV radiation. Chemoprevention of skin cancer using natural agents is suggested as a promising approach and has generated enormous research efforts in recent years. As demonstrated in this study, the compounds of silymarin were readily absorbed by the skin in both in vitro and in vivo experiments. SB showed higher skin absorption than SD and SC. The skin deposition of the non-ionic form was superior to that of ionic molecules. The viable epidermis/dermis represents an important barrier for non-ionic SB permeation via the skin. On the other hand, the stratum corneum is still the predominant permeation barrier for ionic SB. The uptake of silymarin constituents into the skin remained the same after UVB exposure. However, permeation across the skin significantly increased in $\mathrm{pH} 8$ buffer by this irradiation. The preliminary safety examination of the skin showed an acceptable skin tolerance to SB, SD, and SC. This present work indicates the promise of further in vivo and clinical applications of silymarin delivery via the skin. Further study is needed to explore the the delivery vehicles with more-efficient absorption.

\section{Acknowledgements}

We thank the financial support from Chang Gung Memorial Hospital at Keelung (o CMRPG260212).

\section{Author contribution}

Jia-you FANG designed research; Chi-feng HUNG, Yin-ku LIN, and Li-wen ZHANG performed research; Ching-hsien CHANG contributed new analytical tools and reagents; Chifeng HUNG analyzed data; Jia-you FANG wrote the paper.

\section{References}

1 Meeran SM, Katiyar S, Elmets CA, Katiyar SK. Silymarin inhibits UV radiation-induced immunosuppression through augmentation of interleukin-12 in mice. Mol Cancer Ther 2006; 5: 1660-8.

2 Dhanalakshmi S, Mallikarjuna GU, Singh RP, Agarwal R. Dual efficacy of silibinin in protecting or enhancing ultraviolet $B$ radiationcaused apoptosis in HaCaT human immortalized keratinocytes. Carcinogenesis 2004; 25: 99-106.

3 Leiter U, Garbe C. Epidemiology of melanoma and nonmelanoma skin cancer - the role of sunlight. Adv Exp Med Biol 2008; 624: 89-103.

4 Ramos J, Villa J, Ruiz A, Armstrong R, Matta J. UV dose determines key characteristics of nonmelanoma skin cancer. Cancer Epidemiol Biomarkers Prev 2004; 13: 2006-11.

5 Afaq F, Adhami VM, Mukhtar H. Photochemoprevention of ultraviolet B signaling and photocarcinogenesis. Mutat Res 2005; 571: 153-73.

6 Gažák R, Walterová D, Křen V. Silybin and silymarin - new and emerging applications in medicine. Curr Med Chem 2007; 14: 31538.

7 Singh RP, Agarwal R. Flavonoid antioxidant silymarin and skin cancer. Antioxid Redox Signal 2002; 4: 655-63.

8 Pinnel SR. Cutaneous photodamage, oxidative stress, and topical antioxidant protection. J Am Acad Dermatol 2003; 48: 1-22.

9 Yang B, Kotani A, Arai K, Kusu F. Estimation of the antioxidant activities of flavonoids from their oxidation potentials. Anal Sci 2001; 17: 599-604.

10 Zielińska-Przyjemska M, Wiktorowicz K. An in vitro study of the protective effect of the flavonoid silydianin against reactive oxygen species. Phytother Res 2006; 20: 115-9.

11 Gu M, Singh RP, Dhanalakshmi S, Agarwal C, Agarwal R. Silibinin inhibits inflammatory and angiogenic attributes in photocarcinogenesis in SKH-1 hairless mice. Cancer Res 2007; 67: 3483-91.

12 Svobodová A, Zdařilová A, Mališková J, Mikulková H, Walterová D, Vostalová J. Attenuation of UVA-induced damage to human keratinocytes by silymarin. J Dermatol Sci 2007; 46: 21-30.

13 Zhao J, Agarwal R. Tissue distribution of silibinin, the major active constituent of silymarin, in mice and its association with enhancement of phase II enzymes: implications in cancer chemoprevention. Carcinogenesis 1999; 20: 2101-8.

14 Moore JO, Palep SR, Saladi RN, Gao D, Wang Y, Phelps RG, et al. Effects of ultraviolet $B$ exposure on the expression of proliferating cell nuclear antigen in murine skin. Photochem Photobiol 2004; 80: 587-95.

15 Godin B, Touitou E. Transdermal skin delivery: predictions for humans from in vivo, ex vivo and animal models. Adv Drug Deliv Rev 2007; 59: 1152-61.

16 Catz P, Friend D. Transdermal delivery of levonorgestrel: VIII. Effect of enhancers on rat skin, hairless mouse skin, hairless guinea pig skin, and human skin. Int J Pharm 1990; 58: 93-102.

17 Fang JY, Fang CL, Sung KC, Chen HY. Effect of low frequency ultrasound on the in vitro percutaneous absorption of clobetasol 17propionate. Int J Pharm 1999; 191: 33-42.

18 Sloan KB, Wasdo S. Designing for topical delivery: prodrugs can make the difference. Med Res Rev 2003; 23: 763-93.

19 Cole L, Heard C. Skin permeation enhancement potential of aloe vera 
and a proposed mechanism of action based upon size exclusion and pull effect. Int J Pharm 2006; 333: 10-6.

20 Huang ZR, Hung CF, Lin YK, Fang JY. In vitro and in vivo evaluation of topical delivery and potential dermal use of soy isoflavones genistein and daidzein. Int J Pharm 2008; 364: 36-44.

21 Shin SC, Kim HJ, Oh IJ, Cho CW, Yang KH. Development of tretinoin gels for enhanced transdermal delivery. Eur J Pharm Biopharm 2005; 60: $67-71$.

22 Hung CF, Lin YK, Huang ZR, Fang JY. Delivery of resveratrol, a red wine polyphenol, from solutions and hydrogels via the skin. Biol Pharm Bull 2008; 31: 955-62.

23 Meloun M, Burkoňová D, Syrový T, Vrána A. Thermodynamic dissociation constants of silychristin, silybin, silydianin and mycophenolate by the regression analysis of spectrophotometric data. Anal Chim Acta 2003; 486: 125-41.

24 Vávrová K, Lorencová K, Klimentová J, Novotný J, Holý A, Hrabálek A. Transdermal and dermal delivery of adefovir: effects of $\mathrm{pH}$ and permeation enhancers. Eur J Pharm Biopharm 2008; 69: 597-604.

25 Jain AK, Thomas NS, Panchagnula R. Transdermal drug delivery of imipramine hydrochloride. I. Effect of terpenes. J Control Release 2002; 79: 93-101.

26 Panchagnula R, Desu H, Jain A, Kriandarilli S. Effect of lipid bilayer alteration on transdermal delivery of a high-molecular-weight and lipophilic drug: studies with paclitaxel. J Pharm Sci 2004; 93: 2177 83.

27 Touitou E, Godin B, Karl Y, Bujanover S, Becker Y. Oleic acid, a skin penetration enhancer, affects Langerhans cells and corneocytes. J
Control Release 2002; 80: 1-7.

28 Marti-Mestres G, Mestres JP, Bres J, Martin S, Ramos J, Vian L. The "in vitro" percutaneous penetration of three antioxidant compounds. Int J Pharm 2007; 331: 139-44.

29 Batchelder RJ, Calder RJ, Thomas CP, Heard CM. In vitro transdermal delivery of the major catechins and caffeine from extract of Camellia sinensis. Int J Pharm 2004; 283: 45-51.

30 Chang HW, Lai YC, Cheng CY, Ho JL, Ding ST, Liu YC. UV inducibility of rat proliferating cell nuclear antigen gene promoter. J Cell Biochem 1999; 73: 423-32.

31 Athar M, An KP, Morel KD, Kim AL, Aszterbaum M, Longley J, et al. Ultraviolet B (UVB)-induced COX-2 expression in murine skin: an immunohistochemical study. Biochem Biophys Res Commun 2001; 280: 1042-7.

32 Lu C, Lu Y, Chen J, Zhang W, Wu W. Synchronized and sustained release of multiple components in silymarin from erodible glyceryl monostearate matrix system. Eur J Pharm Biopharm 2007; 66: 210-9.

33 Stratton SP, Bangert JL, Alberts DS, Dorr RT. Dermal toxicity of topical (-)epigallocatechin-3-gallate in BALB/c and SKH1 mice. Cancer Lett 2000; 158: 47-52.

34 Bedi MK, Shenefelt PD. Herbal therapy in dermatology. Arch Dermatol 2002; 138: 232-42.

35 Gu M, Dhanalakshmi S, Mohan S, Singh RP, Agarwal R. Silibinin inhibits ultraviolet $B$ radiation-induced mitogenic and survival signaling, and associated biological responses in SKH-1 mouse skin. Carcinogenesis 2005; 26: 1404-13. 